\title{
Migrant blackbirds, Turdus merula, have higher polyunsaturated fatty acids levels in their plasma, but not enhanced susceptibility to peroxidation compared to residents
}

\author{
Johan Kjellberg Jensen ${ }^{1}$, Caroline Isaksson ${ }^{1}$, cas eikenaar ${ }^{2}$, and Martin N. Andersson ${ }^{3}$ \\ ${ }^{1}$ Lund University \\ ${ }^{2}$ Institute of Avian Research \\ ${ }^{3}$ Lund Univ
}

April 28, 2020

\begin{abstract}
Birds have been observed to have dietary preferences for unsaturated fatty acids (FAs) during migration. Polyunsaturated fatty acids (PUFAs) increase the exercise performance of migrant birds; however, PUFAs are also peroxidation prone and might therefore incur increased costs in terms of enhanced oxidative stress in migratory individuals. To shed light on this potential constraint, we analysed plasma FA composition and estimated the susceptibility to peroxidation of migrants and residents of the partially migratory common blackbird (Turdus merula) at a stop-over site during autumn migration. As predicted, migrant birds had higher relative and absolute levels of PUFAs compared to resident birds. This included the strictly dietary $\omega-3$ PUFA $\alpha$-linoleic acid, suggesting a dietary preference for these fatty acids in migrants. Interestingly, the FA unsaturation index, which is an index of lipid peroxidation susceptibility, did not differ between migrants and residents. These findings suggest a mechanism where birds alter their levels of metabolic substrate to increase exercise performance without simultaneously increasing the risk of lipid peroxidation and oxidative stress. In summary, our results are in line with the hypothesis of increased exercise performance being constrained by oxidative stress during migration, which is manifested in changes in the composition of key FAs to retain the unsaturation index constant despite the increased levels of peroxidizable PUFAs.
\end{abstract}

\section{Hosted file}

bb paper final version 1.docx available at https://authorea.com/users/309347/articles/440462migrant-blackbirds-turdus-merula-have-higher-polyunsaturated-fatty-acids-levels-in-theirplasma-but-not-enhanced-susceptibility-to-peroxidation-compared-to-residents 

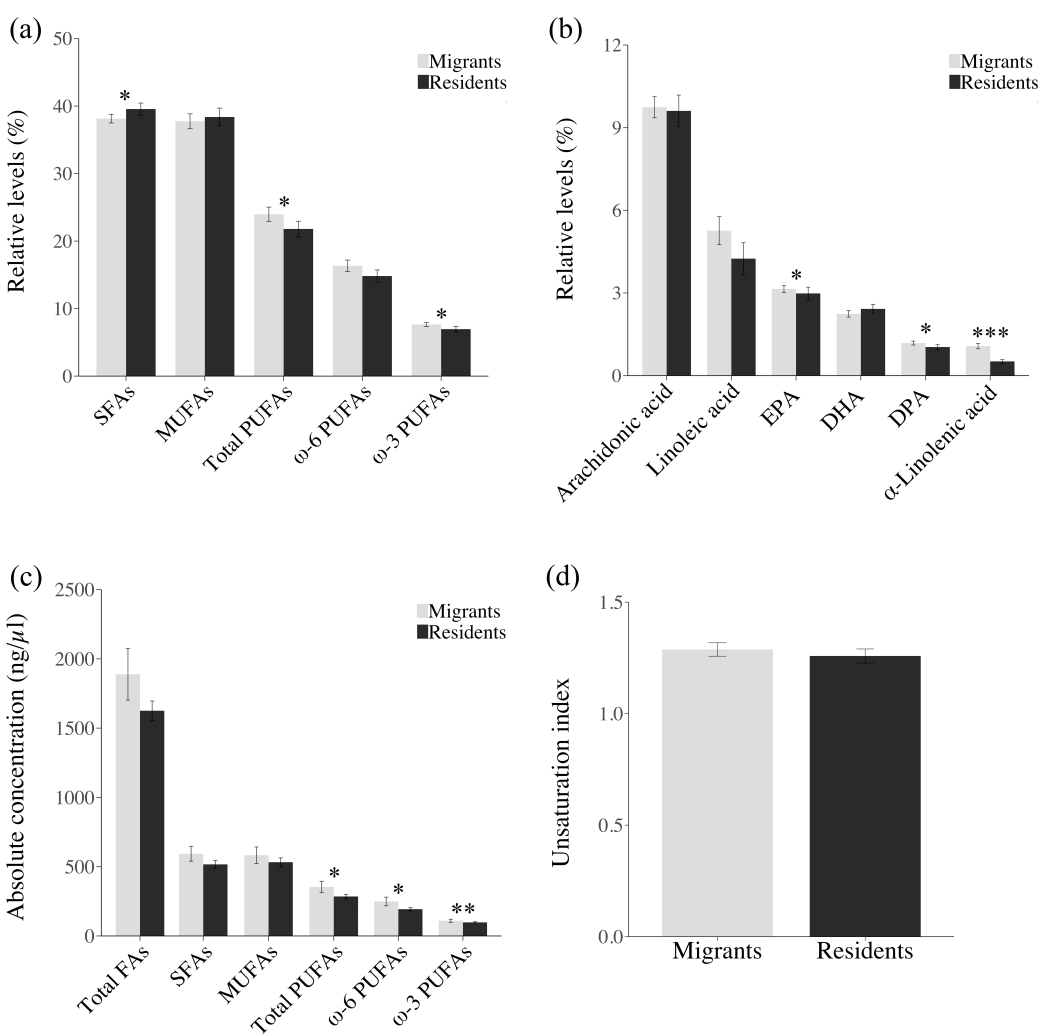
(a)

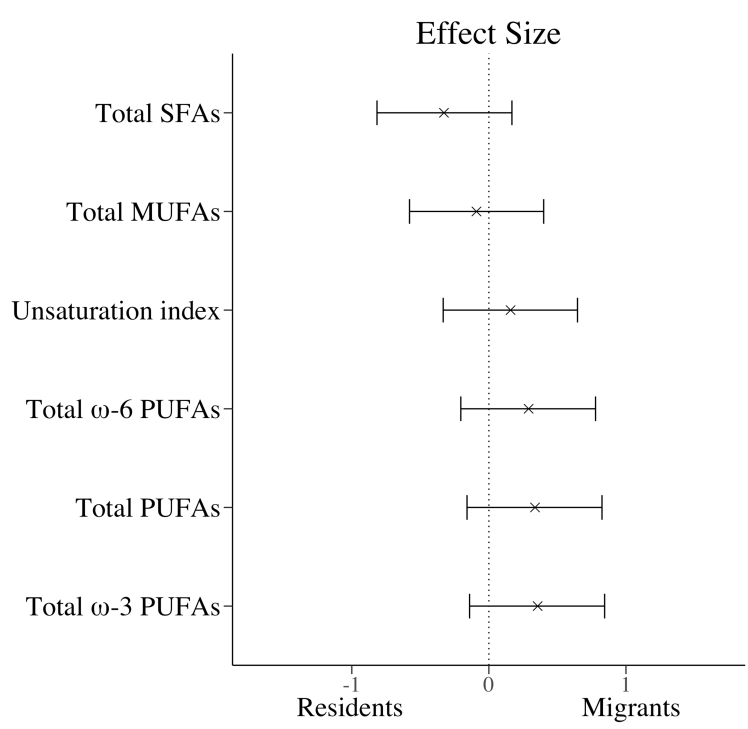

(b)

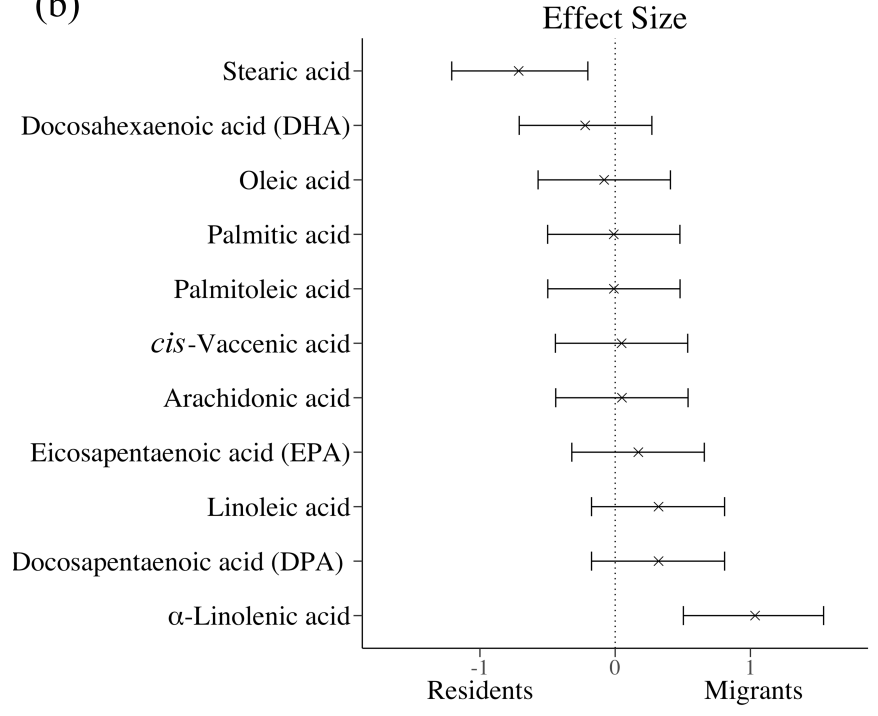

